\title{
STRUCTURAL CHANGES AND MOLECULAR MOBILITY DURING PHYSICAL AGEING OF GLASSES
}

\author{
G.P. Johari \\ Department of Materials Science and Engineering, MaMaster University, \\ Hamilton, Ontario L8S 4L7, Canada
}

\begin{abstract}
Résumé - L'amplitude de la relaxation secondaire et la chaleur spécifique d'un grand nombre de verres dëcroissent au cours des traitements thermiques. Ceci suggère une élimination des régions à forte concentration de volume libre et d'entropie dans les structures. La densification correspondante affecte les propriétés cinétiques, vibrationnelles et électroniques d'un verre de manière différente de ce qui résulte d'une compression. Les régions éliminées correspondent aux centres d'effet tunnel liés au comportement des verres à basse température. Le vieillissement d'un verre fait évoluer sa structure vers celle d'un milieu élastique continu. Ces résultats suggèrent 1 'existence d'une mícrostructure hétérogène.
\end{abstract}

Abstract - The magnitude of secondary relaxation and heat capacity of a variety of glasses decrease on physical ageing. The decrease suggests a collapse of localized high-volume, highentropy regions in its structure. Densification on ageing affects the kinetic, vibrational and electronic properties of a glass differently than does densification on compression. Tunneling centres responsible for the low-temperature behaviour of glasses are identified with the localized regions. Ageing causes a glass to approach a structure with properties of an isotropic elastic continuum. The results support a microscopically heterogeneous structure.

\section{INTRODUCTION}

The change in the structure of a glass on spontaneous densification during its physical ageing may be envisaged in two ways: first, as compaction of homogeneous, coherent, essentially irregular assemblage of molecules, or atoms, capable of passing over quite remote (energy) saddle points in the structure, as for the dense random packing of hard spheres, but in a manner by which the essential elements of local topology of the structure remain unchanged, and, second, as a collapse of lower-density, higher-energy and entropy (or loosely packed) regions, which join together higher-density (and lower-energy) regions in the struture of a glass. The higher-density regions having a tendency to local symmetry which provides a free energy barrier to nucleation and a self-limiting local growth in an apparently heterogeneous structure of a glass.

The excess entropy of a glass /1/, and its secondary relaxation observed below $\mathrm{T}_{\mathrm{g}} / 2 /$, indicate molecular motions in its otherwise rigid matrix. These motions correspond to the thermally activated transitions between configurational states in local, loosely-packed, regions in the structure of a glass and make a substantial contribution to both its thermodynamic and kinetic properties. This paper has two objectives; namely (i) to describe the changes in the number of available configurational states during the physical ageing (annealing, or structural relaxation) of glasses, and (ii) to consider the implications of such changes for our concepts of the structure of a glass and for the existence of local, two-level, states assumed to be responsible for the low-temperature heat capacity, thermal conductivity and dielectric anomalies in the amorphous and other disordered solids.

\section{MOLECULAR MOTIONS AND THERMODYNAMICS}

The occurrence of localized molecular or atomic motions over a time of less than $10^{-3} \mathrm{~s}$ below $\mathrm{T}_{\mathrm{g}}$ is considered intrinsic to the disordered structure of amorphous polymers $/ 3,4 /$, molecular $/ 5,6 /$, network $/ 7 /$ and liquid crystal glasses $/ 8,9 /$ and the glass-like state of orientationally disordered crystals $/ 5,10 /$. Dielectric and mechanical relaxations $/ 7 /$, nmr spectroscopy $/ 11 /$, light scattering $/ 12 /$ and depolarization of fluorescence $/ 13 /$ 
have shown these motions as secondary relaxations which cease to occur on the time scale of one's experiment at $\mathrm{T}<<\mathrm{T}_{\mathrm{g}}$. This means that the configurational state of a glass does not remain fixed on cooling from $\mathrm{T}_{\mathrm{g}}$ to $\mathrm{O}^{\circ} \mathrm{K}$ and the various configurations explored by molecules, or groups of molecules, in an internal thermodynamic equilibrium costitute a degree of freedom, in addition to the vibrational, which determines the thermodynamics of a glass.

A substantial fraction of total expansivity $/ 12,13 /$, heat capacity and entropy $/ 1,14$ / of a glass at $O<\mathrm{T}<\mathrm{T}_{\mathrm{g}}$ is therefore associated with the potential energy of configurational states involved with the thermally activated transitions. The heat capacity of a glass at $T \leq T_{g}$ cannot be entirely described by the Debye theory in which the quantity is determined only by the vibration of atoms or molecules confined to fixed sites in a rigid lattice of a solid treated as an elastic continuum. Since vibrational frequencies intrinsically depend on the energy of configurational states (molecular ensembles of high configurational energy or high volume have low frequency of vibrations and vice versa), the thermodynamics of a glass takes on an additional feature of interest.

\section{MOLECULAR MOTIONS AND THE STRUCTURE OF A GLASS}

Molecular Motions in a glass are usually observed on application of an external stress, which, by biasing them, reveals their existence usually as a peak, or a shoulder in the imaginary component of permittivity or shear modulus. These secondary relaxations are also observed in the equilibrium liquid above $\mathrm{T}_{g}$, along with the main relaxation, in a spectrum measured over a wide frequency range $/ 15 \%$. This represents the accessibility to a liquid of a bimodal spectral distribution of configurational states in the time domain, and this bimodal distribution of orientational diffusion probabilities is unique to a glass or a liquid (in contrast, a crystal has a unimodal distribution). Now if the structure of a glass was truly represented by random closepacked sphere, or a continuous random network model, one expects that the unimodal spectral distribution of a crystal would simply be broadened and shifted in a glass.

We suggest that the occurrence of thermally-activated transitions between configurational states, which contributes also to the volume, entropy and enthaply of a glass, indicates the persistence of stable, local regions in thermodynamic equilibrium in a macroscopically metastable, thermodynamically non-equilibrium state of a glass. Local regions such as these, in a metastable matrix, are known to constitute the structure of certain 'crystalline' solids, namely ice clathrates, which also show a glass-like behaviour/16/. Thus a glass is structurally non- uniform in that a number of presumably statistically distributed regions of low- density and high-entropy, where molecules undergo limited diffusion exist in its structure.

\section{EFFECTS OF PHYSICAL AGEING}

At $\mathbf{T}<\mathbf{T}_{\mathrm{g}}$, a glass is considered to have a fixed configuration which belongs to one of a number of configurations accessible to a liquid at a temperature, $\mathrm{T}_{\mathrm{f}}$, known as the fictive temperature $/ 17 /$. When a glass is held below $\mathrm{T}_{\mathrm{g}}$, its state would irreversibly move into a series of states which correspond to the internal (thermodynamically) equilibrium state of a metastable liquid, or disordered crystal, of decreasing $T_{f}$. During this spontaneous process, the change in the structure of a glass is concomitant with a decrease in its volume, enthalpy and entropy, an increase in the modulus and permittivity and with corresponding changes in the magnitude of its optical (refractive index, attenuation), electronic (semiconducting, band gaps, electron transport etc.), magnetic (Curie point, etc.), and spectroscopic (infrared, Raman, etc.) properties.

The change in the various electrical and mechanical properties during isothermal ageing of a glass is caused by at least four processes, namely:

(i) The contribution to the property from the main relaxation at a temperature and frequency of the secondary relaxation peak decreases. This is because a decrease in volume during ageing of a glass shifts the main relaxation process to a higher temperature and/or a lower frequency.

(ii) A decrease in the height or amplitude of secondary relaxation peak occurs as a consequence of reduction in the number of molecules contributing to the relaxation. This is the case when a decrease in volume is partially due to the collapse of localized high-volume, high-entropy, regions which remain in an internal thermodynamic equilibrium in an otherwise, macroscopically metastable, rigid state of a glass. 
(iii) The magnitude of the property associated with the main relaxation increases with increasing number density of molecules.

(iv) The frequency-independent background loss, both electrical and mechanical, over which the secondary and main relaxations are superposed, decreases with decrease in the volume.

During isothermal ageing, all the four processes occur and cause a change in the property of a glass, but the relative importance of each depends upon the temperature of ageing. This can be determined from measurements of either the isothermal spectrum, or the isochronal temperature variation of a property during or after the ageing period. The criteria used for determining their relative importance in both types of measurements have been given earlier $/ 18,19 /$. The plots in Figs. 1, 2, and 3 show the change in the dielectric and mechanical loss on ageing of four types of glasses. Both the isothermal spectrum and isochrones are given. Our analysis of the dielectric loss data of the quenched and the annealed states of $0.2 \mathrm{NaKO}-0.8 \mathrm{~B}_{2} \mathrm{O}_{3}$ glass obtained by Stevels $/ 21 /$ also showed a decrease in the height of secondary relaxation peak by $\sim 35 \%$ on annealing.
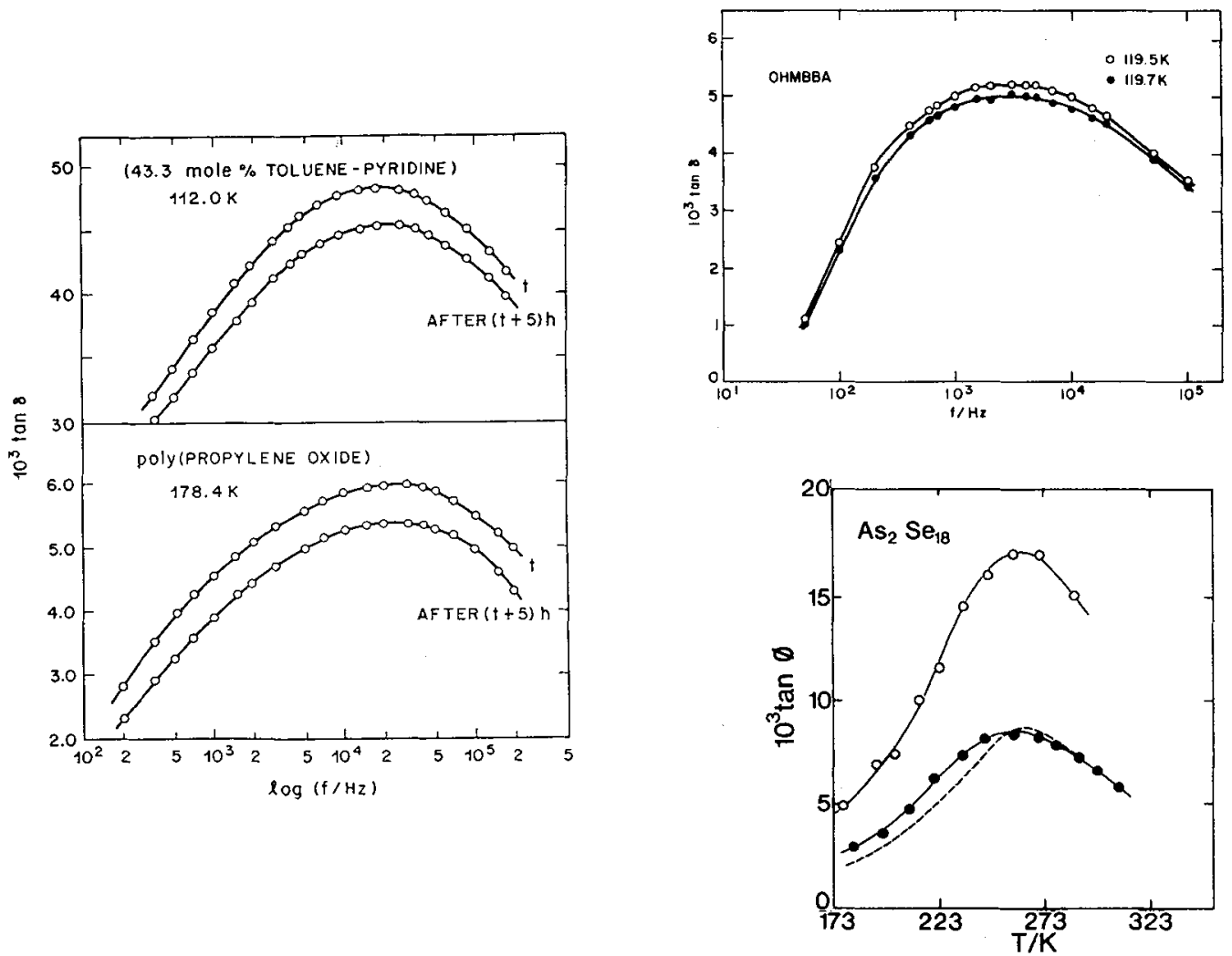

Figure 1 Isothermal spectrum showing a decrease in the dielectric loss tangent on ageing the two types of glasses at the indicated temperatures.

Figure 2 Isothermal spectrum showing a decrease in the dielectric loss tangent of a nematic glass of $O$ hydroxy-p-methoxy butylaniline-p-butylaniline at $119.6 \mathrm{~K}$. Circles are for a sample cooled at $0.4 \mathrm{Ks}^{-1}$ and dots for the same sample heated to $208 \mathrm{~K}$ and cooled at $0.014 \mathrm{Ks}^{-1}$ through its $\mathrm{T}_{\mathrm{g}}$ (204K).

Figure 3 The mechanical loss tangent of $\mathrm{As}_{2} \mathrm{Se}_{18}$ glass $\left(\mathrm{T}_{\mathrm{g}}=348 \mathrm{~K}\right)$ at $1.19 \mathrm{~Hz}$ plotted against temperature. Curve 1 is for a sample quenched from $359 \mathrm{~K}$ to $295 \mathrm{~K}$; 2 for the same sample but annealed at $293 \mathrm{~K}$ for 28 days and 3 for the difference in the loss tangent on ageing (ref. 19). 


\section{DENSIFICATION ON COMPRESSION AND ON AGEING}

Macroscopically, compression has qualitatively the same effect on the volume of a glass as physical ageing. But, in the kinetic properties, which are sensitive to changes at a molecular level, the distinction between the densification on compression and densification on ageing is remarkable. The former causes a shift of the secondary relaxation peak to a lower frequency (or high temperature) by an amount represented as volume of activation, a decrease in its height and an increase in its half-width (whether or not the area under the peak changes is not certain). Thus the rate of secondary relaxation is volume-dependent through the compressibility of a glass. The latter causes no shift of the peak and merely decreases its height and the area under it, as seen in Figs. 1-3. The vibrational frequencies are also affected in remarkably different manners during the two types of densification. Compression, for example, increases the frequency of the fundamental band at $250 \mathrm{~cm}^{-1}$ in selenium glass by $-4 \mathrm{~cm}^{-1} \mathrm{kbar}^{-1}$. Densification on ageing causes no shifts in the frequency of its fundamental $/ 21 /$, first and second harmonics $/ 22 /$.

It is also noteworthy that the Curie point of a metallic glass is decreased by densification on compression /23/ but increased by densification on ageing $/ 24,25 /$.

We conclude that densification on compression decreases the rate of molecular relaxations in qualitatively the same manner as densification on cooling of a glass, but densification on ageing decreases only the number of molecules contributing to the relaxation and not significantly their average rates. Compression undoubtedly decreases uniformly all interatomic or intermolecular distances and the average overall decrease, which can be estimated from the compressibility, is significant. Therefore, the rate of relaxation and the vibrational frequencies change in qualitatively the same manner as on cooling a glass. Ageing, we contend, does not decrease the intermolecular distances in the same manner, but rather by the collapse of the low-density, high-entropy, regions. Since such regions may constitute a substantial volume fraction of a glass, the change in vibrational frequencies is undetectably small. The former type of densification causes an overall decrease in the intermolecular distance much greater than the latter, for the same decrease in volume. The foregoing discussion indirectly supports the hypothesis that a glass has a microscopically heterogeneous structure, for, if the structure was random close-packed or continuous random network, densification by the two procedures would affect qualitatively similar changes in its kinetic, vibrational and electronic properties.

\section{AGEING AND THE TUNNELING STATES}

In a glass, a certain number of molecules or atoms and/or groups of atoms are assumed to have accessible two nearly equivalent equilibrium configurations corresponding to the minima of asymmetric double-well potentials and tunnel between them /26,27/. Tunneling is treated via a model Hamiltonian for two level systems representing the ground states in the two local energy wells and the tunneling states are commonly associated with a small group of atoms undergoing a local rearrangement $/ 26,27 /$. The larger the number of atoms involved, the easier it is to find two ground states of equal energies and therefore, the number of atoms involved is presumed to be small so that the distance between the states in configuration space could be minimized. The zero point entropy of a glass is a measure of the other energetically equivalent regions into whch the glass could have been trapped. These states are mutually inaccessible, because they are distant in configurational space. But mutually accessible nearly degenerate states exist which can tunnel.

If the molecules in the localized regions of relatively loose packing were to be identified as tunneling centres, one would expect three regions of heat capacity behaviour at $\mathrm{O} \leq \mathrm{T} \leq \mathrm{T}_{\mathrm{g}}$, namely, (i) its linear variation with temperature, (ii) its $\mathrm{T}^{3}$ dependence and, (iii) its rapid increase with temperature beyond the $\mathrm{T}^{3}$ variation. This means a change, first from a linear to the Debye behaviour and then a departure from the Debye behaviour at $T \leq T_{g}$ where the degrees of freedom giving rise to secondary relaxations become kinetically unfrozen. The observations of the first behaviour are well documented in the literature $/ 28 /$, and the excess entropy of the glassy solids show the second and third regions $1,29 \%$. Since one consequence of physical ageing is a reduction in the number of such molecular regions, one expects the number of tunneling centres, or the density of localized excitations, to show a corresponding decrease in the heat capacity, dielectric loss and changes in related measurements. Thus a decrease in configurational $\mathrm{Cp}$ at $\mathrm{T} \leq \mathrm{T}_{\mathrm{g}}$ is concommitant with a decrease in the linear term of $\mathrm{C} p$ at $\mathrm{T}<1 \mathrm{~K}$ and with changes in the associated properties, such as thermal conductivity and dielectric loss. This means that a glass would approach the Debye-type behaviour on ageing or on lowering of its fictive temperature.

The above given deduction is in direct contrast with the conclusions of other tunneling models $/ 30,31 /$ which suggest that a high $\mathrm{T}_{\mathrm{g}}$ indicates a small concentration of two-site atoms, or tunneling centres, and therefore 
the amplitude of the $\mathrm{Cp}$ anomaly is less in glasses of high $\mathrm{T}_{\mathrm{g}}$. It is also in contrast with the observation of an approximate inverse relationship between the coefficient of the linear term of $\mathrm{Cp}$ and $\mathrm{T}_{\mathrm{g}}$ of several glasses $132 /$ and, in particular, fictive temperature of vitreous silica $/ 32 /$, though these findings have been recently questioned on grounds of experimental interpretation $/ 33 /$.

A search of literature supports our cunclusions. Densification of silica glass by $\sim 0.1 \%$ on ageing at $1573 \mathrm{~K}$ appreciably decreased its $\mathrm{Cp}$ at $0.5<\mathrm{T}<2 \mathrm{~K} / 34 /$. The same workers also found that, conversely, expansion of $\mathrm{B}_{2} \mathrm{O}_{3}$ glass on thermal treatment (presumably at $\mathrm{T}>\mathrm{T}_{\mathrm{g}}$ ) increased its $\mathrm{Cp}$ by $\sim 10 \%$ at $\mathrm{T} \leq 1 \mathrm{~K} / 35 /$. Densification by both ageing, and by slow cooling of the selenium melt, decreased the $\mathrm{Cp}$ anomaly of its glass /36/ and increased its thermal conductivity /37/. Ageing or annealing of sputtered amorphous films of metallic alloys caused the largest known decrease in their $\mathrm{Cp}$ and increase in thermal conductivity $/ 38 /$. Since the authors $/ 39 /$ independently confirmed that ageing densifies the sputtered amorphons films, we suggest that densification reduced the number of local regions identified here as tunneling centres. Among the above-mentioned glasses, only selenium has been studied in the high temperature region near $\mathrm{T}_{\mathrm{g}}$. Ageing of glassy selenium near $\mathrm{T}_{\mathrm{g}}$ decreased both its $\mathrm{Cp}$ at $\mathrm{T}<2 \mathrm{~K} / 36 /$ and the amplitude of its mechanical relaxation $/ 22 /$.

Although less directly, certain other results in the literature also support the above view. For example, neutron and electron irradiations of synthetic quartz increases the number of atoms involved in localized motions in its crystal and this increases the amplitude of its dielectric relaxation peak $/ 20 \%$ Concomitant with this increase is the appearance of anomalies in its thermal conductivity $/ 40$ / and the low-temperature heat capacity $140,41 /$. Neutron irradiated quartz behaves in this sense like an amorphous solid. The addition of $\mathrm{K}_{2} \mathrm{O}$ to vitreous silica is found to increase both the magnitude of its secondary relaxation $/ 20 /$ and of its lowtemperature anomalous heat capacity, or the density of its localized excitations /33/. Clearly, a procedure that increases the amplitude of secondary relaxation also increases, or causes, the low-temperature anomalous behaviour.

We conclude that ageing causes a glass to approach a structure with the properties of an isotropic elastic continuum. Its heat capacity would tend towards a Debye-behaviour in the entire range of $O<T \leq T_{g}$, when the contributions from the tunneling effects at low temperatures together with the thermally- activated transitions at $\mathrm{T} \leq \mathrm{T}_{\mathrm{g}}$ gradually cease to dominate.

\section{CONCLUSIONS}

The foregoing analyses lead us to conclude as follows:

(i) Localized molecular regions of high-volume and high-entropy exist in an internal thermodynamic equilibrium in an otherwise metastable rigid matrix of a glass at temperatures substantially below $\mathrm{T}_{\mathrm{g}}$. Densification on ageing is partly due to the collapse of such regions. Both the heat capacity and the magnitude of secondary relaxation decrease on ageing.

(ii) The kinetic properties of a glass of a given density would depend upon its thermal history. This means that a description of its kinetics in terms of fictive temperature alone is insufficient.

(iii) The densification on ageing affects the relaxational, vibrational and electronic properties of a glass quite differently than does densification on compression.

(iv) The tunneling centres at $\mathrm{T}<1 \mathrm{~K}$ may be identified as those local regions where thermally-activated molecular motions at $T \leq T_{g}$ occur and contribute to the $C p$ of a glass. If so, the low-temperature heat capacity anomaly is concomitant with the high temperature ( $T \leq T_{g}$ ) non-Debye behaviour. Ageing of a glass decreases both these anomalies. The decrease has been observed in both the heat capacity and dielectric measurements.

\section{ACKNOWLEDGEMENT}

I wish to thank Ecole Superieure de Physique et de Chimie Industrielle de la Ville de Paris, for a term professorship during which this paper was written. I am particularly grateful to Professor Lucien Monnerie for his hospitality during my stay. 


\section{REFERENCES}

/1) Johari, G. P., Phil. Mag. 41 (1980) 41; Ann. N.Y. Acad. Sci. 279 (1976) 102.

$12 /$ Johari, G. P. and Goldstein, M., J. Chem. Phys. $\underline{53}$ (1970) 2372.

/3/ McCrum, N. G., Read, R. E., and Williams, G., Anelastic and Dielectric Effects in Polymeric Solids (Wiley, London 1967).

14/ William, G., in 'Static and dynamic properties of solid polymers' NATO - ASI Proceedings, ed. R. Pethrick and R. W. Richards (D. Reidl, 1982), p. 213.

/5/ Johari. G. P., Ann. N.Y. Acad. Sci. 279 (1976) 117.

16/ Johari, G. P., in "Plastic Deformation in Amorphous and semicrystalline materials" Les Mouches Lectures 1982 (Les Editions de Physique, 1982), p. 109.

/7/ Perez J., Cavaille, J. Y., Etienne, S., Fouquet, F., and Guyot, F. Ann. Phys. (Fr.) 8 (1983) 417.

/8/ Johari, G. P., Phil. Mag. $\underline{46}$ (1982) 549; J. Chem, Phys. 77 (1982) 5165.

/9/ Johari, G. P., Goodby, J. W. and Johnson, G. E. Nature 297 (1982) 315.

/10/ Pathmanathan, K. and Johari, G. P., J. Phys. C (1985) in press.

/11/ McCall, D. W., Nat. Bur. Std. (U.S.) Special Publi. 301 (1969) 475.

/12/ Patterson, G. D., J. Phys. Chem. 89 (1985) 1344.

/13/ Leduc, J. and Kalantar, A. H., J. Chem. Phys. 73 (1980) 5330.

/14/ Goldstein, M., Ann. N.Y. Acad. Sci. 279 (1976) 68.

/15/ Johari, G. P., J. Chem. Phys. $\underline{58}$ (1973) 1766.

/16. Johari, G. P. and Chew, H. A. M., Phil. Mag. B 49 (1984) 281.

/17/ Tool, A. Q., J. Am. Ceram. Soc. 29 (1946) 240.

/18/ Johari, G. P., J. Chem. Phys. 77 (1982) 4619.

19/ Etienne, S., Cavaille, J. Y., Perez, J. and Johari, G. P., Phil. Mag. 51A (1985) L35.

/20/ Stevens, J. M., J. Non-Cryst. Solids, 40 (1980) 69.

/21/ Stephens, R. B., J. Appl. Phys. 49 (1978) 5855.

122/ Etienne, S., Thesis (1985).

/23/ Bouzabata, B., Ingalls, R. and Rao, K. V., J. Appl. Phys. 53 (1982) 2324.

124/ Chen, H. S., Sherwood, R. C., Leamy, H. J., and Georgy, E. M., IEEE Trans. Magn. 12 (1976) 933.

/25/ Libermann, H. N., Graham, C. D. and Flanders, P. J., ibid 13 (1977) 1541.

/26/ Anderson, P. W., Halperin, B. I., and Varma, C. M., Philos. Mag. 25 (1972) 1.

127 Phillips, W. A., J. Low Temp. Phys. 7 (1972) 351.

/28/ Bhattacharya, A., Contemp. Phys. 22 (1981) 117.

/29/ Johari, G. P., J. Chim. Phys. 82 (1985) 283.

130. Cohen, M. H. and Grest, G. S., Phys. Rev. Lett. 45 (1980) 1271.

/31/ Phillips, J. C., Phys. Rev. B. 24 (1981) 1744.

/32/ Raychaudhari, A. K. and Pohl, R. O., Solid State Commun. 37 (1980) 105; Phys. Rev. B.25 (1982) 1310.

133/ McDonald, W. C., Anderson, A. C. and Schroeder, J., Phys. Rev. B. 31 (1985) 1090.

/34/ Vandorpe, M., Lasjaunias, J. C. and Penn, G., J. Non-Cryst. Solids, $\underline{57}$ (1983) 157.

135/ Lasjaunias, J. C., Penn, G., Ravex, A., and Vandorpe, M., J. Physique, L41 (1980) L131; J. Non-Cryst. Solids 57 (1983) 157.

136/ Lasjaunias, J. C., Picot, B., Ravex, A., and Vandorpe, M., The Second Conference of the Condensed Matter Division of the European Physical Society on Dielectrics an Phonons, Budapest 1974, p. 199.

137/ Calemczuk, R., Thse, Docteur des Sciences Physique, Université de Grenoble (1983), p. 133.

138/ Lasjaunias, J. C., Ravex, A., Laborde, D. and Bethoux, O., Phyica 126B (1984) 126.

139/ Ravex, A., Lasjaunias, J. C. and Bethoux, O., Solid State Commun. $\underline{40}$ (1981) 853 .

140/ Berman, R., Proc. Roy. Soc. (London), A208 (1951) 90.

141/ Gardner, J. W., and Anderson, A. C., Phys. Rev. B23 (1981) 474. 